\title{
A machine learning based forecast model for the COVID-19 pandemic and investigation of the impact of government intervention on COVID-19 transmission in China
}

\author{
Xingcheng Lu \\ The Hong Kong University of Science and Technology \\ Dehao Yuan \\ The Hong Kong University of Science and Technology \\ Wanying Chen \\ The Hong Kong University of Science and Technology \\ Jimmy Fung ( $\square$ majfung@ust.hk) \\ The Hong Kong University of Science and Technology
}

\section{Research Article}

Keywords: COVID-19, Baidu Index, Machine Learning, Prediction, Intervention

Posted Date: September 10th, 2020

DOl: https://doi.org/10.21203/rs.3.rs-73671/v1

License: (c) (i) This work is licensed under a Creative Commons Attribution 4.0 International License.

Read Full License 


\section{A machine learning based forecast model for the COVID-19 pandemic and investigation of the impact of government intervention on COVID-19 transmission in China}

9 Abstract

10 The coronavirus disease 2019 (COVID-19) pandemic has killed over 0.3 million people, disrupted people's 11 normal lives, and severely restricted economic activities globally. In this work, a model for the next-day

12 COVID-19 prediction in China was built based on the ensemble back-propagation neural network machine 13 learning technique, Baidu migration index, internal travel flow index, and confirmed cases from the 14 previous days. The 10 -fold cross-validation results showed that the model performs well in estimating the next-day confirmed cases with a correlation coefficient of 0.97 . To investigate the impacts of government interventions on the spread of this new coronavirus infection, the Baidu migration index and internal travel

17 flow index multiplied by a factor of two were input into the trained machine learning model, and the results showed that the confirmed cases in the analyzed cities would increase dramatically. The correlation between the daily new confirmed cases and some meteorological factors were also analyzed, and the results revealed that these factors are not dominant in influencing the spread of this disease. Overall, the results of this work suggest that besides early diagnosis and medical treatment, a city lockdown policy is one of the most

22 effective methods in suppressing the rapid spread of COVID-19.

23 Keywords: COVID-19, Baidu Index, Machine Learning, Prediction, Intervention 


\section{Introduction}

31 In December 2019, several cases of unidentified viral pneumonia were observed in Wuhan, China (Lu et al., 2020). Later, the patients were identified to have been affected by a new coronavirus. As the disease spread with an unprecedented speed over China, on January 30, 2020, the World Health Organization (WHO) officially declared the outbreak of "2019 novel coronavirus (2019-nCoV)" as a "public health emergency of international concern" (Sohrabi et al., 2020). The coronavirus disease 2019 (COVID-19) has been detrimental not only to China but the whole world (Wang et al., 2020), and as of Aug 4, 2020, over 18.2 million people globally have been reported to be infected by this serious disease (Worldmeter, 2020). Around 0.692 million deaths were confirmed to be due to 2019-nCoV infection, and the mortality rate of this disease has reached 3.8\%. Unfortunately, no miracle drug or vaccine has yet been found or developed after 7 months since this disease was first identified. As the most crucial black swan event of 2020, the COVID-19 pandemic has severely restricted the global economy and has sharply reduced goods consumption in some countries (Fernandes, 2020). Most social and commercial activities have been banned in many Western countries, such as Italy, Spain, and the U.S.A. (Nicola et al., 2020). This pandemic has dragged China's GDP growth into the worst recession since 1990 (Kishan, 2020); in the first quarter of 2020, China's economy shrank 6.8\% compared with the previous year (Tan and Cheng, 2020).

Because of this significant disruption caused by the COVID-19 pandemic, it is crucial to identify the key factors affecting the infectiousness of 2019-nCoV and develop a reliable model for near-real-time forecasting (Petropoulos and Makridakis, 2020, Wynants et al., 2020). Since the wider outbreak of this disease in January, substantial efforts have been devoted globally to unveil its mysteries (Fanelli and Piazza, 2020, Wu et al., 2020, Yang et al., 2020). Using the Glob al Epidemic and Mobility Model, a study reported that the travel restrictions in Wuhan on January 23, 2020, had marked effects on reducing the spread of the new coronavirus infection globally, with an $80 \%$ importation reduction until mid-February (Chinazzi et al., 2020). Based on a dynamic transmission model, public health interventions were found to be effective in reducing the risk of 2019-nCoV spread (Su et al., 2020). Based on the regression method, a study found that, without the lockdown in Wuhan, the infected cases in other Hubei cities, excluding Wuhan, would have increased by $52.6 \%$ on February 29 (Fang et al., 2020). Studies have also reported that airborne transmission might play a role in the spread of this novel coronavirus (Liu et al., 2020). The RNA of 2019nCoV could be detected in an air sample obtained from a patients' toilet at the Fangcang Hospital in Wuhan. However, in the analyzed patient rooms, the concentration of airborne 2019-nCoV was quite low or undetectable. Meteorological factors have also been reported to affect the transmission of this disease in the environment. Based on the reported cases in China from January 20 to February 29, 2020, 2019-nCoV 

However, due to the limitation of the sample sizes and interference from other confounding factors, the impacts of environmental factors (e.g., aerosol concentration and temperature) on the 2019-nCoV transmission have not yet been confirmed.

To date, only close-quarters interaction with infected person has been confirmed as a major factor for 2019nCoV transmission (Pung et al., 2020). Thus, population dynamics should be an important factor in constructing a COVID-19 pandemic forecast model. Baidu, established in 2000, is a Chinese technology company specializing in its search engine, Internet services, and artificial intelligence (https://www.baidu.com/). Baidu Migration, a big data visualization project developed by Baidu Inc., is utilized to display the population migration of Chinese cities. Baidu Migration is based on the LBS

2 (Location-Based Service) open platform and Baidu Tianyan to calculate and analyze the track and characteristics of population migration in China through the inbound and outbound migration of inter-city and the intensity of intracity traffic. Baidu Migration provides historical and near-real-time indexes to quantify the cross-city population migration and within-city population movement in the major cities of China. In this work, a prediction model for the next-day 2019-nCoV-infected cases was built based on the Baidu migration data and ensemble back-propagation neural network (BPNN) machine learning technique. The city lockdown policy implemented in China is already known to be a highly important factor in controlling the spread of 2019-nCoV in China (Zhang et al., 2020). Some previous researches have perceived the fruitfulness of travel restriction in controlling the spread of COVID-19 (Fanelli and Piazza, 2020, Ray et al., 2020, Sardar et al., 2020). As distinguished from previous studies, this paper combines the BPNN model with Baidu Migration LBS data and considers the impact of intracity and inter-city travel on the spread of the disease, so it has specific promotion and generalization ability. This work further investigated the effectiveness of this policy by enlarging the Baidu migration index and input it into the trained BPNN model. 


\section{Baidu Migration Index}

The Baidu migration index can be downloaded at https://qianxi.baidu.com/. This index is based on the Baidu Maps LBS open platform, which tracks and collects the location information of the users via mobile phones. The data include the migration index in a single day from cities other than the specific city (based on the cross-city travel population number), the percentage of migrant population coming from each of the other cities, and the internal travel flow index within the specific city (based on the population travel within the city and the number of local residents). According to previous studies, the mean incubation period of 2019-nCoV is approximately 5-6 days (Backer et al., 2020, Linton et al., 2020). Therefore, in this work, the Baidu migration data of the previous 4-7 days' averages have been used for the prediction of the confirmed cases in the current day. A previous epidemic estimation study also applied this index to analyze the COVID-19 outbreak in China (Li et al., 2020). Before inputting the data into the BPNN model, the movement data (migration from other cities, internal movement, and the percentage of migrant population from other cities) together with the averages of the reported confirmed cases in the previous 4-7 days were processed using Equations (1) and (2) for the "external_risk" and "internal_risk" calculations:

$$
\begin{aligned}
& \text { external_risk }(t, C)=\frac{1}{4} \sum_{i=t-7}^{i=t-4} Q R\left(t_{i}, C\right) \cdot \sum_{a \in \text { all-other-cities } X Z\left(t_{i}, a\right) \cdot Q X\left(t_{i}, a, C\right)} \\
& \text { internal_risk }(t, C)=\frac{1}{4} \sum_{\substack{i=t-4 \\
i=t-4}} L D\left(t_{i}, C\right) \cdot X Z\left(t_{i}, C\right)
\end{aligned}
$$

where $Q R(t, C)$ is the migration index (dependent on the total migrant population) from other cities to the targeted city $C$ on day $t, X Z(t, a)$ represents the number of new confirmed cases in the city $a$ on day $t, Q X$ $(t, a, C)$ is the percentage of the migrant population in city $C$ that came from city $a$ on day $t, L D(t, C)$ is the internal travel flow index for city $C$ on day $t$, and $X Z(t, C)$ is the number of new reported confirmed cases in the targeted city $C$ on day $t$. Besides Wuhan, many Chinese cities began to report COVID-19 cases after January 23, 2020 (Leung et al., 2020). The growth rate of the disease in most of the cities approached zero after February 24. In early March, imported infection cases from foreign countries were observed in some Chinese cities. Therefore, the data used for analysis in this work ranged from January 23 to March 5, 2020. The data from the previous 4-7 days were needed as input for the prediction. Hence, the prediction period was from January 30 to March 5, 2020, giving a total of 36 days. The top 100 cities with the highest number of accumulated confirmed cases (except Wuhan) till early March were obtained. The names of these 100 cities, averages of the migration index and internal travel flow index, and the numbers of the confirmed case by March $5^{\text {th }} 2020$ are shown in Table S1. The origin of the novel coronavirus has not yet been determined. Snake, bat, and pangolin are all possible hosts of this new virus (Ji et al., 2020, Lam et al., 
2020, Zhou et al., 2020). Thus, besides interactions with patients, there might be some other causative factors for the spread of this infection in Wuhan, the city of origin, such as contact with possible animal hosts. Therefore, the analysis for Wuhan was not included in this work. But if people migrated from Wuhan to other cities, the 'external_risk' contributed by Wuhan was considered.

\section{BPNN machine learning technique}

Backward Propagation Neural Network (BPNN) BPNN is a machine learning model which can regress non-linear relations between input features and output targets. The framework of BPNN is a composition of several layer transformations. Each layer transformation is the composition of a nonlinear activation function and a linear transformation. The known linear activation is user-defined and is usually chosen to be hyperbolic tangent function or ReLU function (return itself if input $>0$ and 0 if input $<0$ ). The linear transformation can be written as the matrix multiplication, where the entries of the matrices can be adjusted to minimize the prediction loss. The algorithm that is used to learn the learnable parameters is gradient descent. Each parameter is adjusted by its gradient with respect to the loss, multiplied by a user-defined constant (called learning rate). The number of learnable parameters is determined by the number of layers, and the neuron numbers of each layer. The greater number of learnable parameters a network equips, the more complicated function the network can model. The detail of the BPNN is shown in the following formulae.

Step 1: Compute the network output.

$$
\hat{Y}=W_{n_{\text {out }} \times n_{n}} \times\left[\text { layer }_{n} \circ \text { layer }_{n-1} \circ \cdots \circ \text { layer }_{1} \circ \text { layer }_{0}(X)\right]+b_{n_{\text {out }} \times 1}
$$

where layer $r_{k}(Z)=g\left(W_{n_{k} \times n_{k-1}} \times Z+b_{n_{k} \times 1}\right)$, g is a non-linear activation function.

Step 2: Compute the prediction loss.

$$
\text { loss }=\sum|\widehat{Y}-Y|^{2}
$$

Step 3: For every weight and bias, update them according to the loss. The $\alpha$ is the user-defined constant, called learning rate.

$$
\begin{gathered}
W=W-\alpha \cdot \frac{\partial \operatorname{los} s}{\partial W} \\
b=b-\alpha \cdot \frac{\partial \operatorname{los} s}{\partial b}
\end{gathered}
$$


BPNN Ensemble Due to the random initialization of the learnable parameters, and the random split of training/test sets, the single BPNN training may harbor relatively large uncertainty. Ensemble method, which refers to the aggregation of several BPNNs, can effectively improve the stability of the BPNN predictions. The usual ways of conducting BPNN ensemble are computing the simple or weighted average of different BPNNs, splitting the training/test sets in a more systematic way (in our case, 10-fold cross validation, which will be introduced later). Since we want a more accurate estimation of the confirmed cases trend, we conducted the BPNN ensemble to reduce the error due to the randomness of BPNNs.

An ensemble BPNN was used to model the relationship between the external_risk/internal_risk on the fourth to the seventh preceding days and the number of new confirmed cases on the current day. The number of days since January 23, 2020 was also input into the BPNN for training. Before being input into the BPNN, external_risk, internal_risk and the number of days since January 23, 2020 were normalized to values between 0 and 1. The structure of the ensemble BPNN and processing method is displayed in Fig. 1. Briefly, each BPNN component consists of two layers with five and three neurons, respectively. The data from 90 cities were extracted randomly and input into five BPNNs for the training step (Step-1). The data from the other 10 cities were input into the built-up model for the verification step (Step-2). The 10-fold crossvalidation results were then obtained after conducting this process 10 times (Step-3). The 10-fold crossvalidation ensures each city to have a chance to be tested. This can eliminate the errors caused by the random split of training/test sets. As the weights in the BPNNs were assigned randomly, the results may not be consistent in different rounds of training. Hence, the above three steps were processed 100 times to estimate the uncertainty of the results caused by the random weight initialization in the BPNN.

\section{Results}

\section{Prediction performance of the BPNN}

A comparison of the estimated and observed numbers of COVID-19 cases (10-fold cross-validation) by March 5, 2020 for 100 analyzed Chinese cities is shown in Fig. 2 (a). Please note that the verifications presented in Figure 2 are based on the hold-out testsets in the 10-fold cross validation. From the total case number perspective, the BPNN performed well, generally yielding reasonable estimations before March 5 for most cities $(\mathrm{R}=0.97)$. However, the total number of confirmed cases in some cities was underestimated by the BPNN. For example, in Ezhou, 1,394 confirmed cases were observed by March 5, but the BPNN underestimated this number by $51 \%$, with a prediction of 688 cases. In Xiaogan, the numbers of observed and estimated confirmed cases were 3,518 and 2,478 respectively, an underprediction of $29.6 \%$. In Suizhou, the BPNN underestimated the number of confirmed cases by 232, a discrepancy of $17.8 \%$. The migration index and within-city population movement index can be taken to represent the overall degree of mobility 
in a city. However, they do not contain information on gatherings of large groups. Even if only one infected person had attended such a gathering, this might have led to an outbreak within a cluster of people. This may be one of the main reasons for the underestimation of the confirmed COVID-19 cases in these three cities in Hubei province.

Fig. 2(b) shows the cumulative numbers of estimated and observed confirmed COVID-19 cases in the 100 analyzed cities from January 30 to March 5, 2020. The BPNN was trained 100 times. The green line in the figure is the median value of the estimated cumulative case numbers, with the shaded area representing two standard deviations. In general, the BPNN accurately predicted the trend and magnitude of the confirmed cases from January 30 to March 5. However, a relatively large discrepancy appeared from February 10 to February 17. For example, the BPNN underestimated the number of confirmed COVID-19 cases on February 15 by $2.8 \%$ (721 cases). The discrepancy between the prediction and observation narrowed after February 25, and the percentage difference dropped below 1\% for March 5. This demonstrates that this ensemble BPNN can accurately capture the relationship between internal_risk/external_risk on the fourth to the seventh preceding days and the number of new confirmed cases on the current day. This in turn indicates that population movement is a determinant of the transmission of this new virus. However, this ensemble BPNN model cannot be used to predict the importation of cases into China from other countries, because no inbound flight information was included in the training input.

\section{Impacts of population movement}

Based on information released by the Ministry of Transport of the People's Republic of China, population movement during the 2020 Chinese Spring Festival (January 10 to February 18, 2020) decreased by 50\% relative to 2019 (http://www.mot.gov.cn/jiaotongyaowen/202002/t20200220_3334989.html, in Chinese). Hence, the Baidu migration index and within-city travel flow index were multiplied by a factor of two and input into the trained ensemble BPNN to estimate the impacts that greater population movement would have had on COVID-19 transmission. As shown in Fig. 3, after the Baidu migration index and the withincity travel flow index were multiplied by two, the number of estimated COVID-19 cases was much higher than the official confirmed count. The rate of increase of reported COVID-19 infections began to drop on February 12 and approached zero after February 21. However, if the migration index and within-city travel flow index had been double their actual values, the infection rate would not have begun to slow until February 20, and the estimated number of infected cases would have continued to rise even after March 5. On March 5, the number of estimated COVID-19 cases in this counterfactual scenario reached 127,275 (two standard deviations: 57,961-237,467), which is 4.6 times larger than the actual reported number of confirmed cases $(27,494)$ for the 100 analyzed cities. These results indicate that the city lockdown policy after January 23 in a number of Chinese cities was highly effective in controlling the wide spread of the 
novel coronavirus. Although the lockdown policy interrupted most economic activities, it proved worthwhile, as many residents' lives were saved and substantial medical costs were avoided.

The impact of government intervention on COVID-19 infection cases in six major Chinese cities, Beijing $\left(39.90^{\circ} \mathrm{N}, 116.41^{\circ} \mathrm{E}\right.$; capital city), Shanghai $\left(31.23^{\circ} \mathrm{N}, 121.47^{\circ} \mathrm{E}\right.$; eastern China), Guangzhou $\left(23.13^{\circ} \mathrm{N}\right.$, 113.26 ${ }^{\circ}$; southern China), Chongqing $\left(29.43^{\circ} \mathrm{N}, 106.91^{\circ} \mathrm{E}\right.$; southwestern China), Chengdu $\left(30.57^{\circ} \mathrm{N}\right.$, $104.07^{\circ} \mathrm{E}$; southwestern China) and Nanjing $\left(32.06^{\circ} \mathrm{N}, 118.80^{\circ} \mathrm{E}\right.$; eastern China), is presented in Fig. 4. Without the implementation of the city lockdown policy, the number of confirmed COVID-19 cases in these six cities would have shot up to 2,237 (946-4,554), 1,904 (445-4,490), 825 (306-1,782), 3,708 (1,914-5,592), 1,768 (304-4,442) and 418 (174-690), respectively, by March 5, 2020. Considering the actual numbers of confirmed cases, the government interventions for these cities were highly effective, and the rate of new infections approached zero after February 12. However, it was estimated that without these interventions, the number of COVID-19 cases would have continued to increase after March 5. This demonstrates that reducing personal social contact (e.g., staying at home and maintaining social distance) was one of the main factors responsible for the successful reduction of COVID-19 transmission in China after mid-February. It should be noted that a relatively large uncertainty exists in the values estimated for the six cities here. Besides migration intensity, coronavirus transmission is also controlled by group gatherings and disinfection measures in the community, but neither of these factors was input into the BPNN due to a lack of data. However, our results do imply that the number of COVID-19-infected cases would have increased drastically without appropriate control of population movement.

\section{Discussion}

Based on our results, population movement is a key determinant of the transmission of COVID-19. The ensemble BPNN designed in this work can be combined with the Baidu migration index and within-city travel flow index to predict near-future COVID-19 infection. For the regions that do not have the Baidu migration index, population movement data can be used as the alternative for the infection prediction. However, several other factors also play important roles in suppressing the transmission of the disease, such as self-quarantine, personal testing for the SARS-CoV-2 virus, face mask wearing and disinfection measures in the community. Fig. 5 shows the Baidu search indices for "face mask" and "disinfectant" in the six major cities listed above. Both indices were relatively high for January 23 to February 5, which implies that Chinese citizens became strongly motivated to protect themselves from this novel disease by wearing face masks and using disinfectant when the lockdown of Wuhan city was announced on January 23. As the reported number of confirmed COVID-19 cases began to drop after February 10, the search indices for "face mask" and "disinfectant" decreased gradually as well. Some recent works have pointed out that face mask use can effectively curtail the community transmission of COVID-19 (Cheng et al., 2020, 
Eikenberry et al., 2020). Without face mask wearing and effective disinfection, it is likely that the number of confirmed COVID-19 cases would have risen to a higher level under the same degree of population movement. Therefore, to make the ensemble BPNN proposed in this work more universal and better able to predict COVID-19 transmission in other countries, variables that describe face mask wearing and disinfection measures need to be included.

It is still unclear whether meteorological factors, such as temperature, relative humidity $(\mathrm{RH})$ and wind speed, influence COVID-19 transmission. Fig. 6 shows the correlation between the number of daily confirmed cases (on day $t$ ) and the averages on the fourth to the seventh preceding days for related meteorological factors (from day $t-7$ to day $t-4$ ). The correlation coefficients between the number of new daily confirmed cases and the day $t-7$ to day $t-4$ averages of temperature, $\mathrm{RH}$, wind speed and $\mathrm{RH}$ $\times$ temperature were $-0.044,0.055,-0.036$ and -0.043 , respectively. No clear correlation was found between the number of confirmed cases and any of these meteorological factors, which is similar to the conclusions made by a previous work (Yao et al., 2020).

As the numbers of new daily confirmed cases are mainly governed by population migration and inter-city travel intensity, these numbers were normalized by "internal_risk $\times$ external_risk" to remove the impact of population contact on COVID-19 transmission, as shown in Equation (3):

$$
\text { NCase }=\frac{X Z(t, C)}{\frac{1}{4} \sum_{i=t-7}^{i=t-4}\left(\text { internal }_{r} i s k\left(t_{i}, C\right) * \text { external }_{r} i s k\left(t_{i}, C\right)\right)}(3)
$$

As shown in Table 1, the correlation coefficients between the meteorological factors and the normalized number of new daily confirmed cases ranged from -0.0642 to -0.00727 . No clear relationships were found between COVID-19 transmission and the meteorological factors even after removing the risk factor of personal contact. Two other extreme conditions were also considered. First, if the number of confirmed cases in the target city was much higher than that in other cities, only internal_risk should be taken into account. Second, if the number of confirmed cases in the target city was much smaller than that in other cities, only external_risk should be considered. However, when the number of new confirmed cases was also normalized by internal_risk and external_risk, respectively, still no clear relationship was found between COVID-19 transmission and the meteorological factors, as shown in Table 1. This is consistent with the conditions and spread of the disease in other countries. For example, 100-500 new cases of COVID-19 were reported during June to July everyday in Singapore, a tropical region, where the temperature is generally over $30^{\circ} \mathrm{C}$. However, this does not necessarily mean that the transmission of the novel coronavirus is not influenced by temperature and RH. Influenza viruses are known to be affected by 
temperature and humidity. Meteorological factors may simply have less influence on the transmission of

274 this novel coronavirus than face mask wearing and government intervention.

\section{Conclusions}

276 Since the COVID-19 outbreak in late January, this disease has now spread globally and has substantially 277 disrupted people's lives and economic activities. In this work, an ensemble BPNN machine learning model combining with Baidu migration index was developed to predict near future COVID-19 cases in the major cities over China. The forecast performance is satisfactory and can be used to predict the transmission of COVID-19 in other regions. Our results indicate that restricting people's movement is one of the main factors contributing to the control of this disease. Thus, government interventions are likely the main reason for the successful control over the pandemic by mid-February in most Chinese cities (except Wuhan). In some other countries, such as Italy, although initially the numbers of infected cases are high, the daily confirmed cases began decreasing gradually once the lockdown restrictions were implemented (Sebastiani et al., 2020). Therefore, besides early diagnosis and medical treatment, restricting the population movement and maintaining social distance appear to be the best methods to suppress the spread of this highly contagious virus when no effective vaccine is yet available. Some previous studies have pointed out that meteorological factors can also influence 2019-nCoV transmission. However, based on our analysis, these factors are not dominant in controlling the transmission rate of this disease. That being said, although meteorological factors are not dominant in influencing the COVID-19 pandemic, we cannot rule out the possibility that these factors still influence the spread of this coronavirus to a limited extent. More studies are warranted to further understand the meteorological impacts on this new disease.

\section{Acknowledgement}

This work was supported by the Research Grants Council of Hong Kong Government (Project No. T24/504/17. The authors declare no competing financial interest.

(1)

(1)

99

0

01

02

3




\section{References:}

305 Backer JA, Klinkenberg D, Wallinga J. Incubation period of 2019 novel coronavirus (2019-nCoV) 306 infections among travellers from Wuhan, China, 20-28 January 2020. Eurosurveillance $307 \quad 2020 ; 25(5): 2000062$.

308 Cheng VC, Wong S-C, Chuang VW, So SY, Chen JH, Sridhar S, et al. The role of community-wide wearing 309 of face mask for control of coronavirus disease 2019 (COVID-19) epidemic due to SARS-CoV-2. Journal 310 of Infection 2020.

311 Chinazzi M, Davis JT, Ajelli M, Gioannini C, Litvinova M, Merler S, et al. The effect of travel restrictions 312 on the spread of the 2019 novel coronavirus (COVID-19) outbreak. Science 2020;368(6489):395-400.

313 Eikenberry SE, Mancuso M, Iboi E, Phan T, Eikenberry K, Kuang Y, et al. To mask or not to mask: 314 Modeling the potential for face mask use by the general public to curtail the COVID-19 pandemic. 315 Infectious Disease Modelling 2020.

316 Fanelli D, Piazza F. Analysis and forecast of COVID-19 spreading in China, Italy and France. Chaos, 317 Solitons \& Fractals 2020;134:109761.

318 Fang H, Wang L, Yang Y. Human mobility restrictions and the spread of the novel coronavirus (2019-ncov) 319 in china. National Bureau of Economic Research; 2020.

320 Fernandes N. Economic effects of coronavirus outbreak (COVID-19) on the world economy. Available at 321 SSRN 35575042020.

322 Ji W, Wang W, Zhao X, Zai J, Li X. Cross-species transmission of the newly identified coronavirus 2019323 nCoV. Journal of medical virology 2020;92(4):433-40.

324 Kishan H. China's first-quarter economic hit from coronavirus looking more severe: Reuters poll; 2020. 325 Available from: https://www.reuters.com/article/us-china-economy-poll/china-first-quarter-economic-hit326 from-coronavirus-looking-more-severe-reuters-poll-idUSKBN20T00L. [Accessed 4 Aug 2020].

327 Lam TT-Y, Jia N, Zhang Y-W, Shum MH-H, Jiang J-F, Zhu H-C, et al. Identifying SARS-CoV-2-related 328 coronaviruses in Malayan pangolins. Nature 2020:1-4.

329 Leung K, Wu JT, Liu D, Leung GM. First-wave COVID-19 transmissibility and severity in China outside 330 Hubei after control measures, and second-wave scenario planning: a modelling impact assessment. The 331 Lancet 2020. 
Li C, Chen LJ, Chen X, Zhang M, Pang CP, Chen H. Retrospective analysis of the possibility of predicting the COVID-19 outbreak from Internet searches and social media data, China, 2020. Eurosurveillance 2020;25(10):2000199.

Linton NM, Kobayashi T, Yang Y, Hayashi K, Akhmetzhanov AR, Jung S-m, et al. Incubation period and other epidemiological characteristics of 2019 novel coronavirus infections with right truncation: a statistical analysis of publicly available case data. Journal of clinical medicine 2020;9(2):538.

Liu Y, Ning Z, Chen Y, Guo M, Liu Y, Gali NK, et al. Aerodynamic characteristics and RNA concentration of SARS-CoV-2 aerosol in Wuhan hospitals during COVID-19 outbreak. BioRxiv 2020.

Lu H, Stratton CW, Tang YW. Outbreak of pneumonia of unknown etiology in Wuhan, China: The mystery and the miracle. Journal of medical virology 2020;92(4):401-2.

Nicola M, Alsafi Z, Sohrabi C, Kerwan A, Al-Jabir A, Iosifidis C, et al. The socio-economic implications of the coronavirus pandemic (COVID-19): A review. International journal of surgery (London, England) 2020;78:185.

Petropoulos F, Makridakis S. Forecasting the novel coronavirus COVID-19. PloS one 2020;15(3):e0231236.

Pung R, Chiew CJ, Young BE, Chin S, Chen MI, Clapham HE, et al. Investigation of three clusters of COVID-19 in Singapore: implications for surveillance and response measures. The Lancet 2020.

Ray D, Salvatore M, Bhattacharyya R, Wang L, Du J, Mohammed S, et al. Predictions, role of interventions and effects of a historic national lockdown in India's response to the COVID-19 pandemic: data science call to arms. Harvard data science review 2020;2020(Suppl 1).

Sardar T, Nadim SS, Rana S, Chattopadhyay J. Assessment of Lockdown Effect in Some States and Overall India: A Predictive Mathematical Study on COVID-19 Outbreak. Chaos, Solitons \& Fractals 2020:110078.

Sebastiani G, Massa M, Riboli E. Covid-19 epidemic in Italy: evolution, projections and impact of government measures. European journal of epidemiology 2020;35(4):341.

Shi P, Dong Y, Yan H, Li X, Zhao C, Liu W, et al. The impact of temperature and absolute humidity on the coronavirus disease 2019 (COVID-19) outbreak-evidence from China. MedRxiv 2020.

Sohrabi C, Alsafi Z, O’Neill N, Khan M, Kerwan A, Al-Jabir A, et al. World Health Organization declares global emergency: A review of the 2019 novel coronavirus (COVID-19). International Journal of Surgery 2020. 
Su L, Hong N, Zhou X, He J, Ma Y, Jiang H, et al. Evaluation of the secondary transmission pattern and epidemic prediction of COVID-19 in the four metropolitan areas of China. Frontiers in Medicine 2020;7.

Tan H, Cheng E. China says its economy shrank by $6.8 \%$ in the first quarter as the country battled coronavirus; 2020. Available from: https://www.cnbc.com/2020/04/17/china-economy-beijing-contractedin-q1-2020-gdp-amid-coronavirus.html. [Accessed 8 Aug 2020].

Wang C, Horby PW, Hayden FG, Gao GF. A novel coronavirus outbreak of global health concern. The Lancet 2020;395(10223):470-3.

Worldmeter. COVID-19 CORONAVIRUS PANDEMIC; 2020. Available from: https://www.worldometers.info/coronavirus/. [Accessed 4 Aug 2020].

Wu JT, Leung K, Leung GM. Nowcasting and forecasting the potential domestic and international spread of the 2019-nCoV outbreak originating in Wuhan, China: a modelling study. The Lancet 2020;395(10225):689-97.

Wynants L, Van Calster B, Bonten MM, Collins GS, Debray TP, De Vos M, et al. Prediction models for diagnosis and prognosis of covid-19 infection: systematic review and critical appraisal. bmj 2020;369.

Yang Z, Zeng Z, Wang K, Wong S-S, Liang W, Zanin M, et al. Modified SEIR and AI prediction of the epidemics trend of COVID-19 in China under public health interventions. Journal of Thoracic Disease 2020;12(3):165.

Yao Y, Pan J, Liu Z, Meng X, Wang W, Kan H, et al. No Association of COVID-19 transmission with temperature or UV radiation in Chinese cities. European Respiratory Journal 2020;55(5).

Zhang C, Chen C, Shen W, Tang F, Lei H, Xie Y, et al. Impact of population movement on the spread of 2019-nCoV in China. Emerging Microbes \& Infections 2020;9(1):988-90.

Zhou P, Yang X-L, Wang X-G, Hu B, Zhang L, Zhang W, et al. A pneumonia outbreak associated with a new coronavirus of probable bat origin. nature 2020;579(7798):270-3. 
389

390

391

392

393

394

395

396

397

398

399

400

401

402

403

92

93

98

99

00

01

02

Table 1: Correlations between the daily confirmed cases normalized by the risk factors of the previous 4-7 days' average and meteorological factors.

\begin{tabular}{ccccc}
\hline Normalized by & Temperature & RH & Wind Speed & Temperature $\times$ RH \\
\hline internal_risk $\times$ external_risk & -0.0605 & -0.0642 & -0.0073 & -0.0614 \\
internal_risk & -0.0435 & -0.0099 & -0.0156 & -0.0428 \\
external_risk & -0.0561 & -0.0510 & -0.0043 & -0.0546 \\
\hline
\end{tabular}

94

5

6




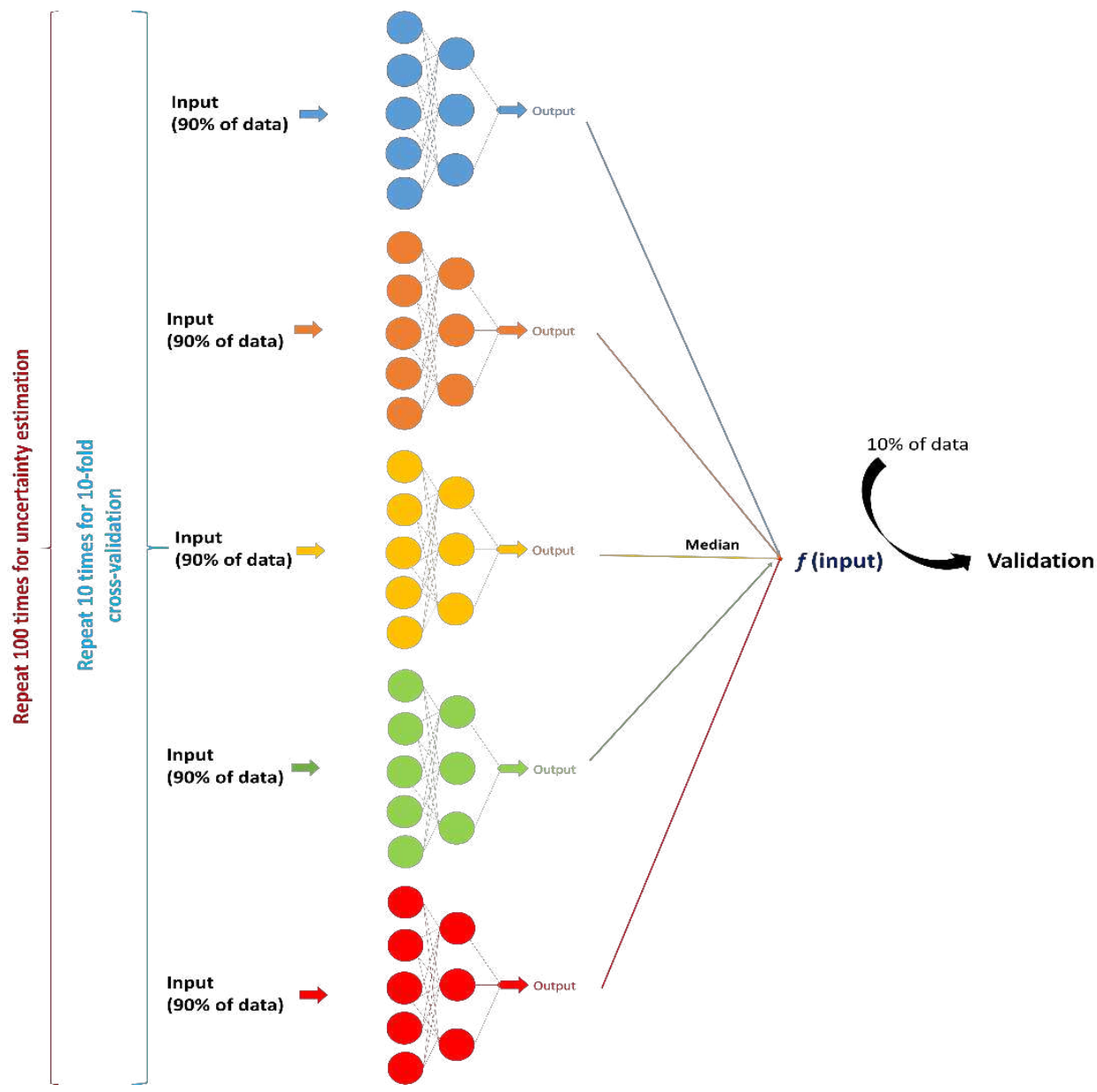

Fig. 1: Structure of the ensemble BPNN.

407

408 

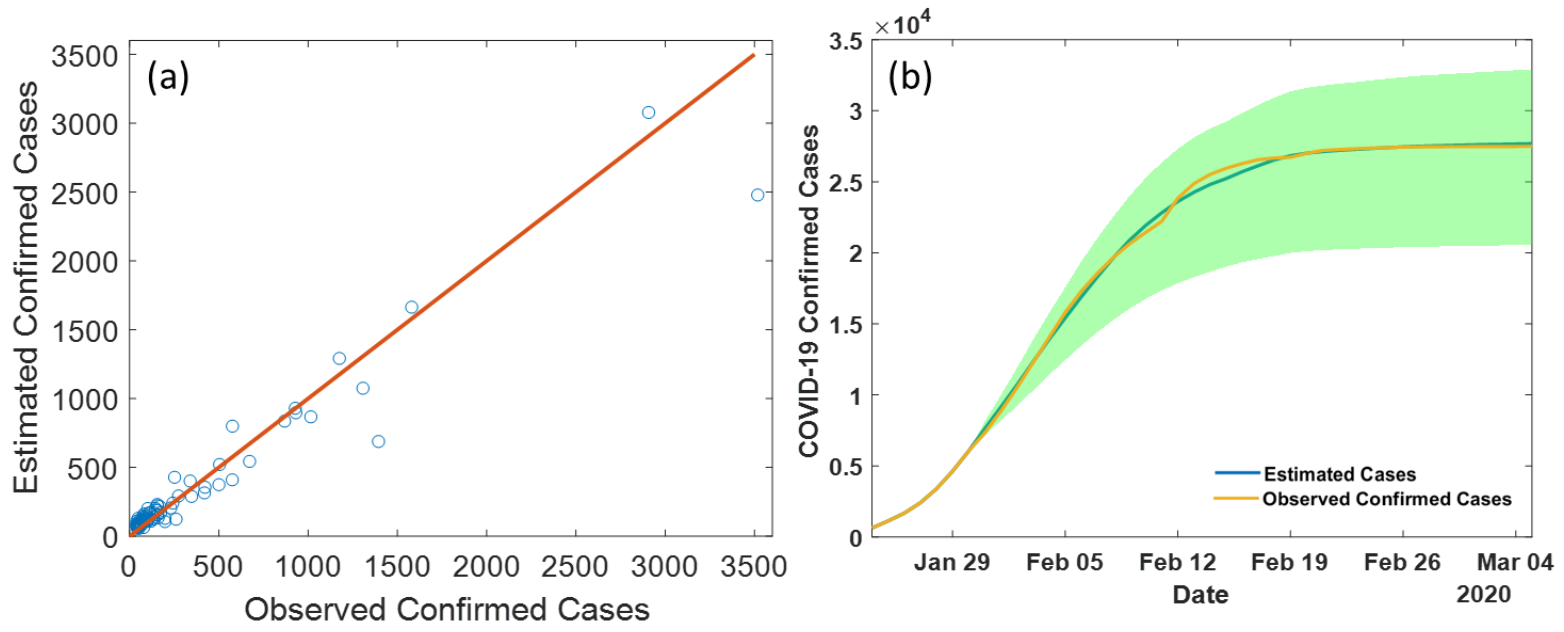

Fig. 2: (a) Comparison between the total observed confirmed cases and estimated confirmed cases on

411 March 5, 2020; (b) comparison between the observed and estimated accumulated cases. The green shaded area is within two standard deviations of running the ensemble BPNN 100 times. 


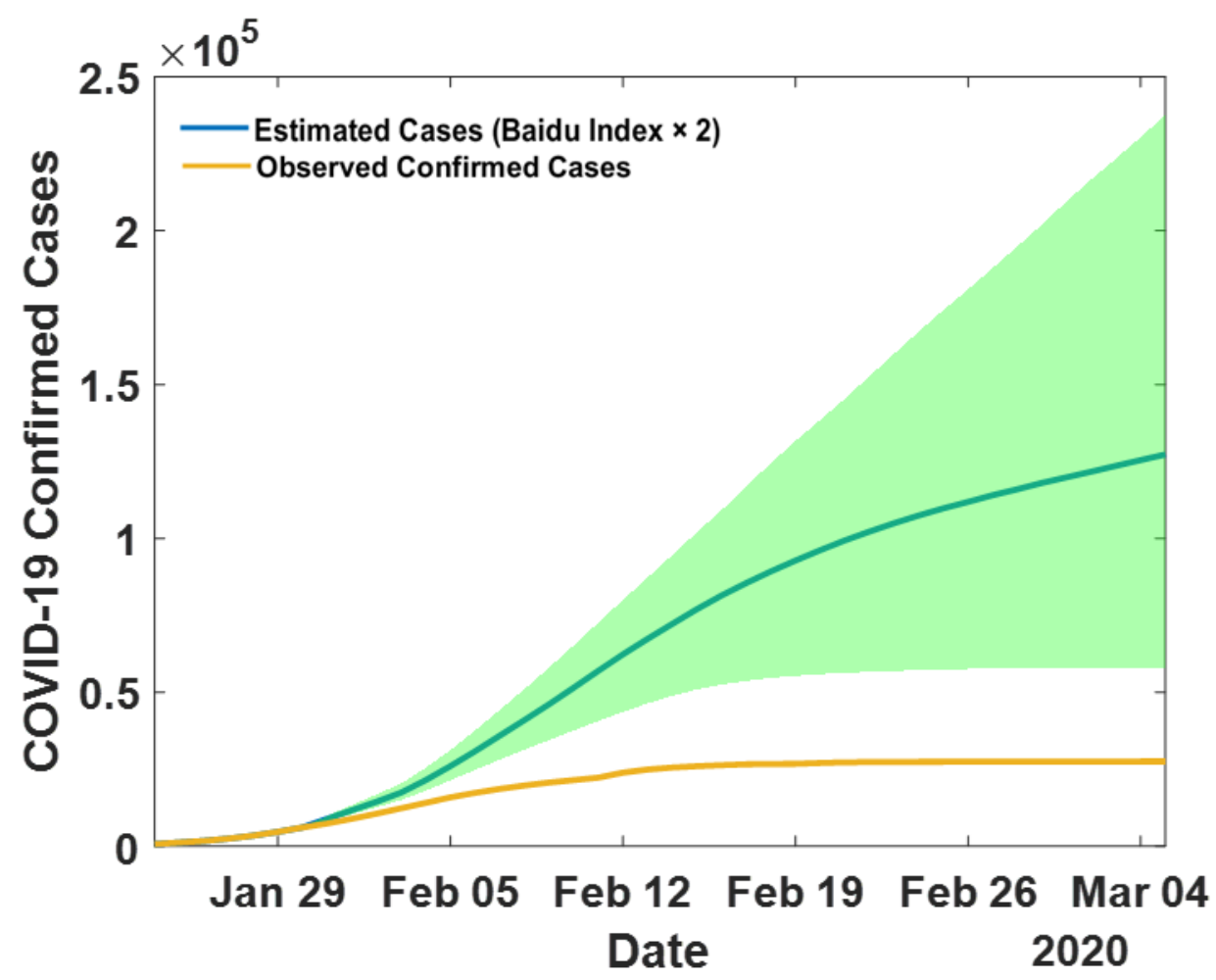

429 Fig. 3: Estimated number of COVID-19 cases after doubling the Baidu indices for the 100 analyzed cities. 

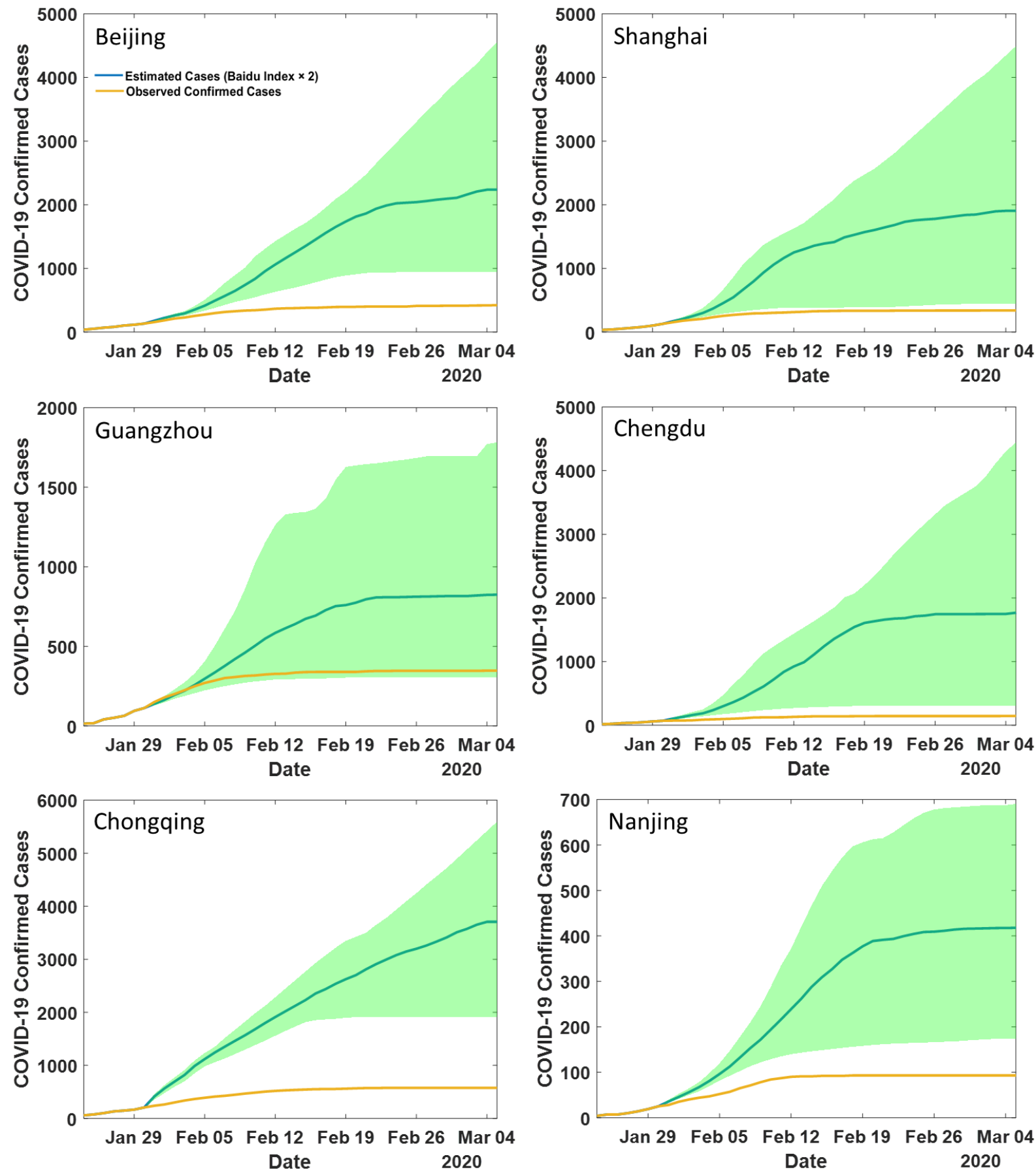

Fig. 4: Estimated number of COVID-19 cases after doubling the Baidu indices for six major cities in 

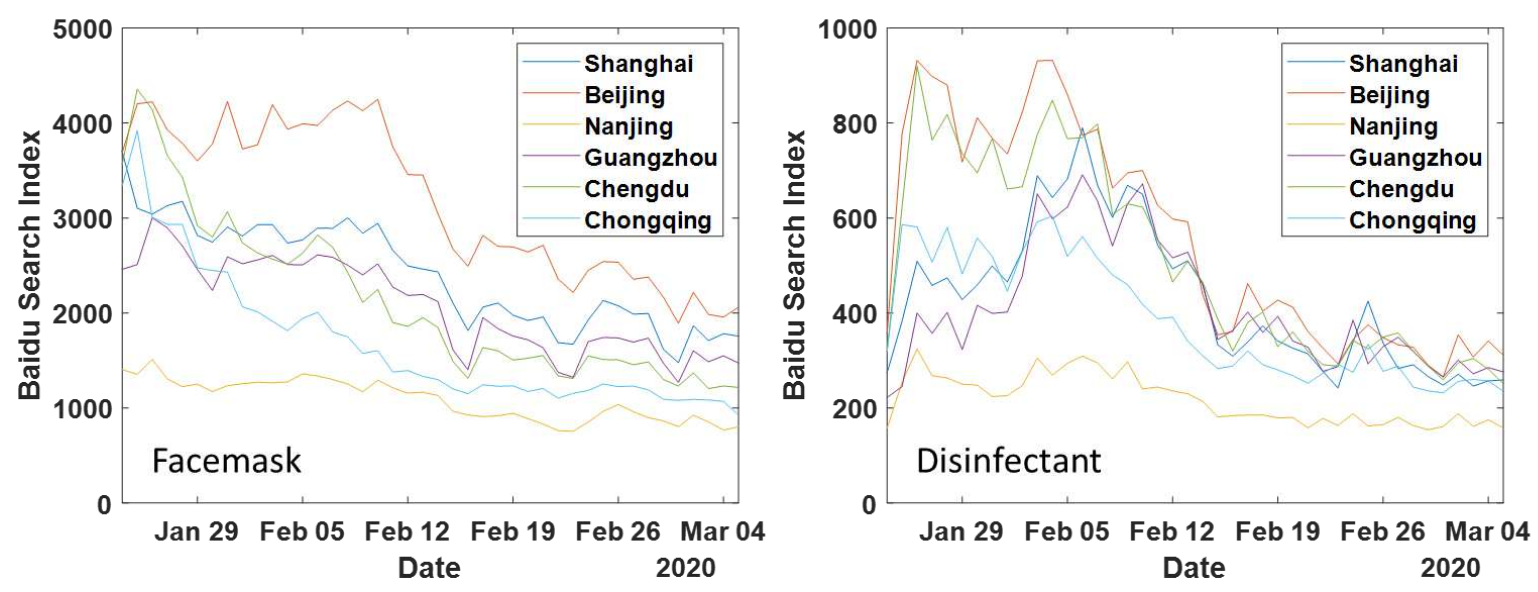

Fig. 5: Baidu search indices for "face mask" and "disinfectant" for six major cities in China. 

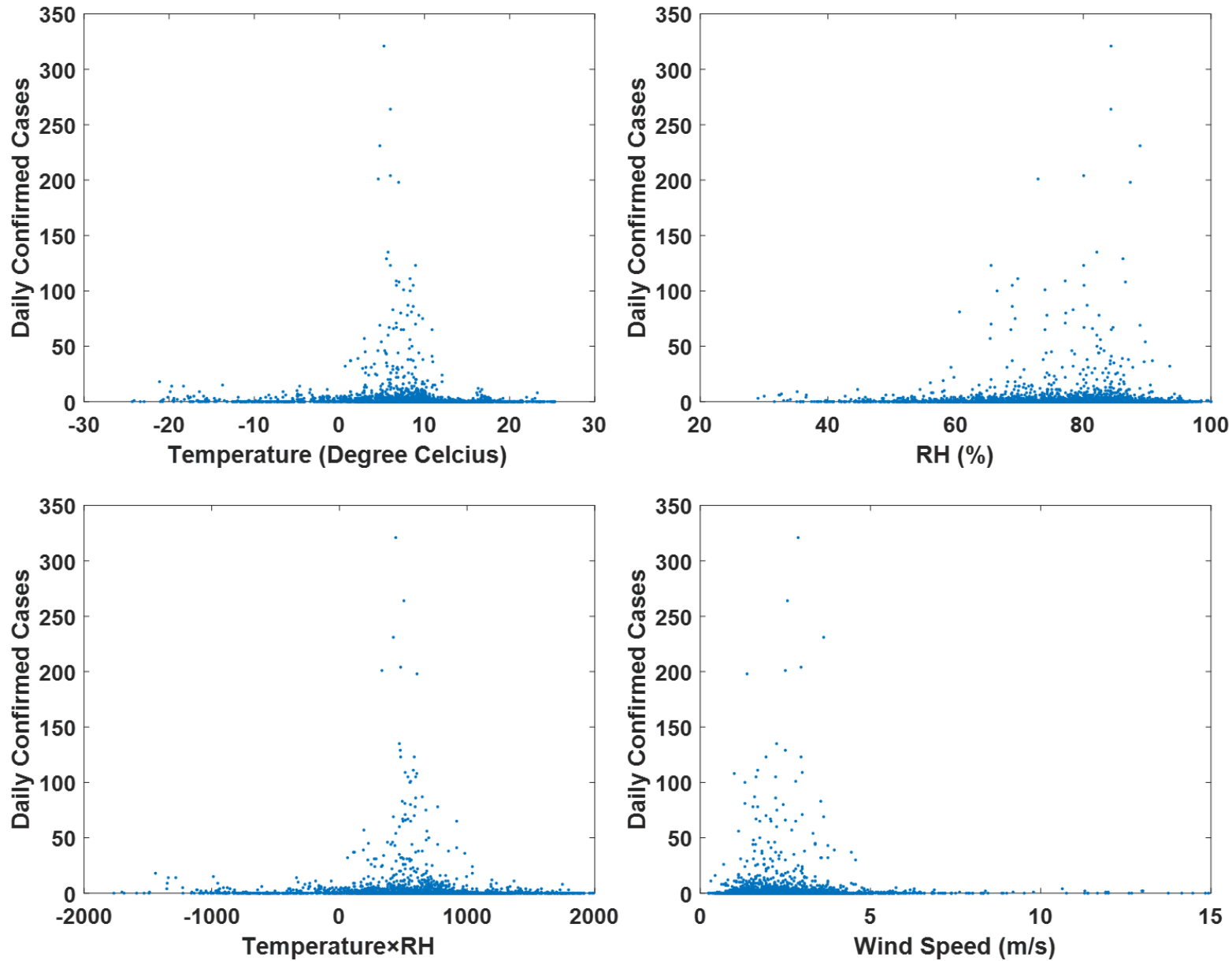

461 Fig. 6: Relationships between the daily confirmed cases and meteorological factors (previous 4-7 days' 462 averages). 
Figures

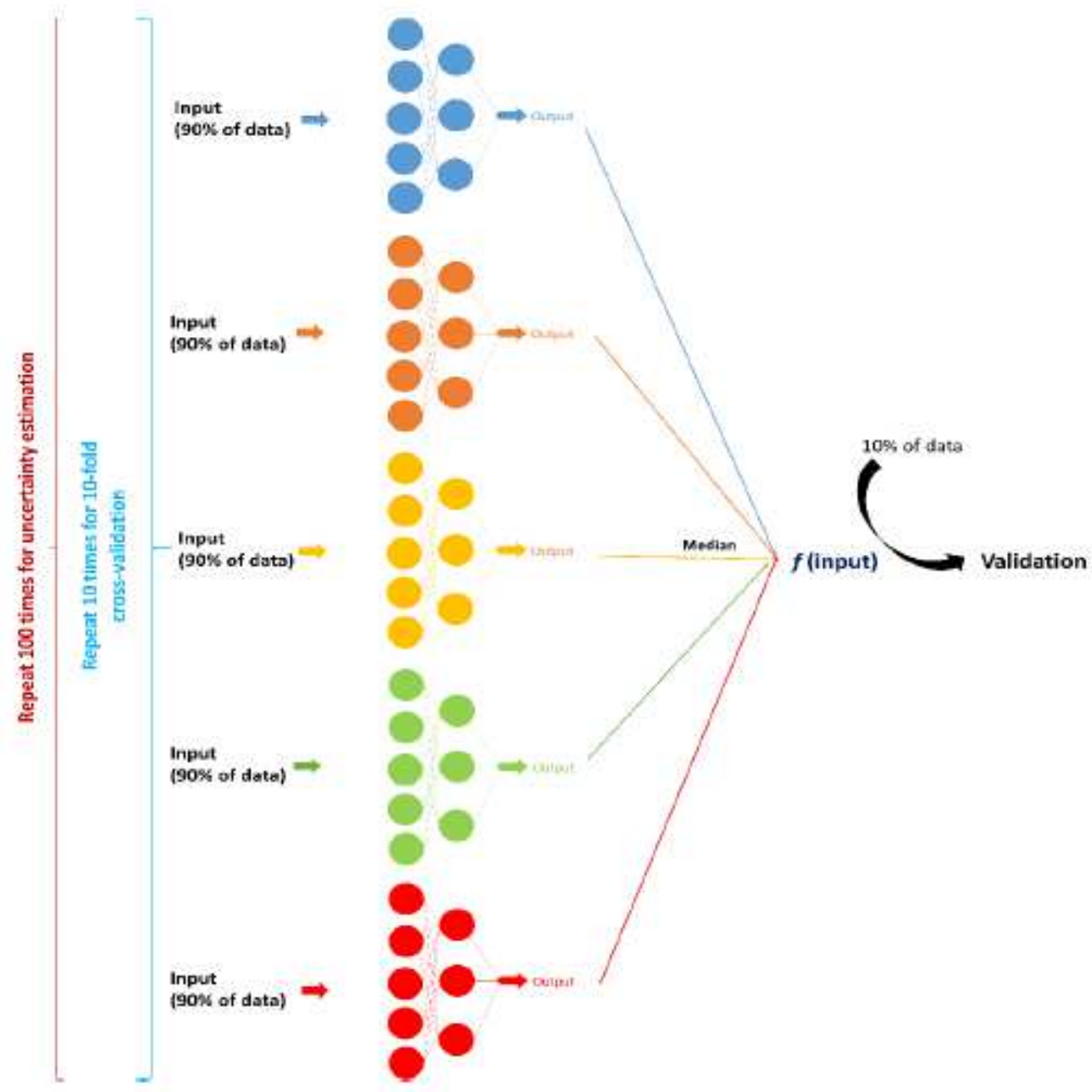

Figure 1

Structure of the ensemble BPNN. 

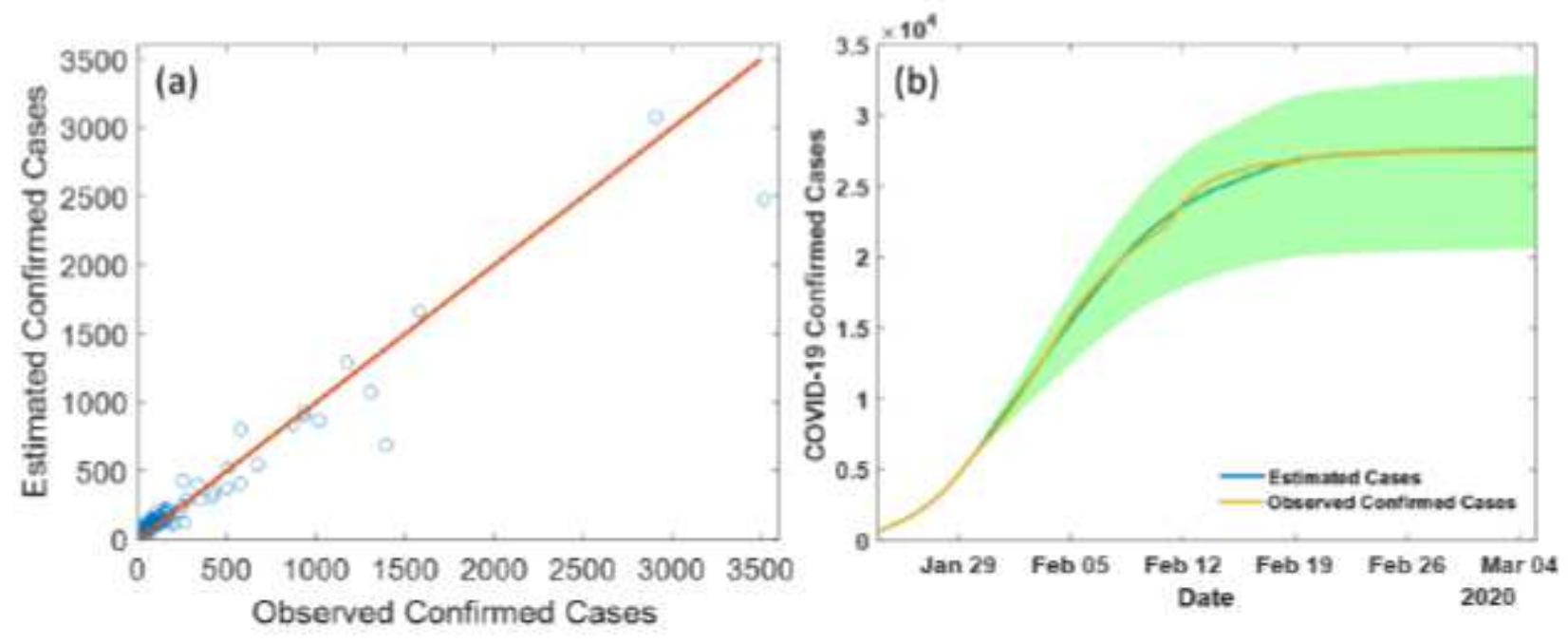

Figure 2

(a) Comparison between the total observed confirmed cases and estimated confirmed cases on March 5, 2020; (b) comparison between the observed and estimated accumulated cases. The green shaded area is within two standard deviations of running the ensemble BPNN 100 times.

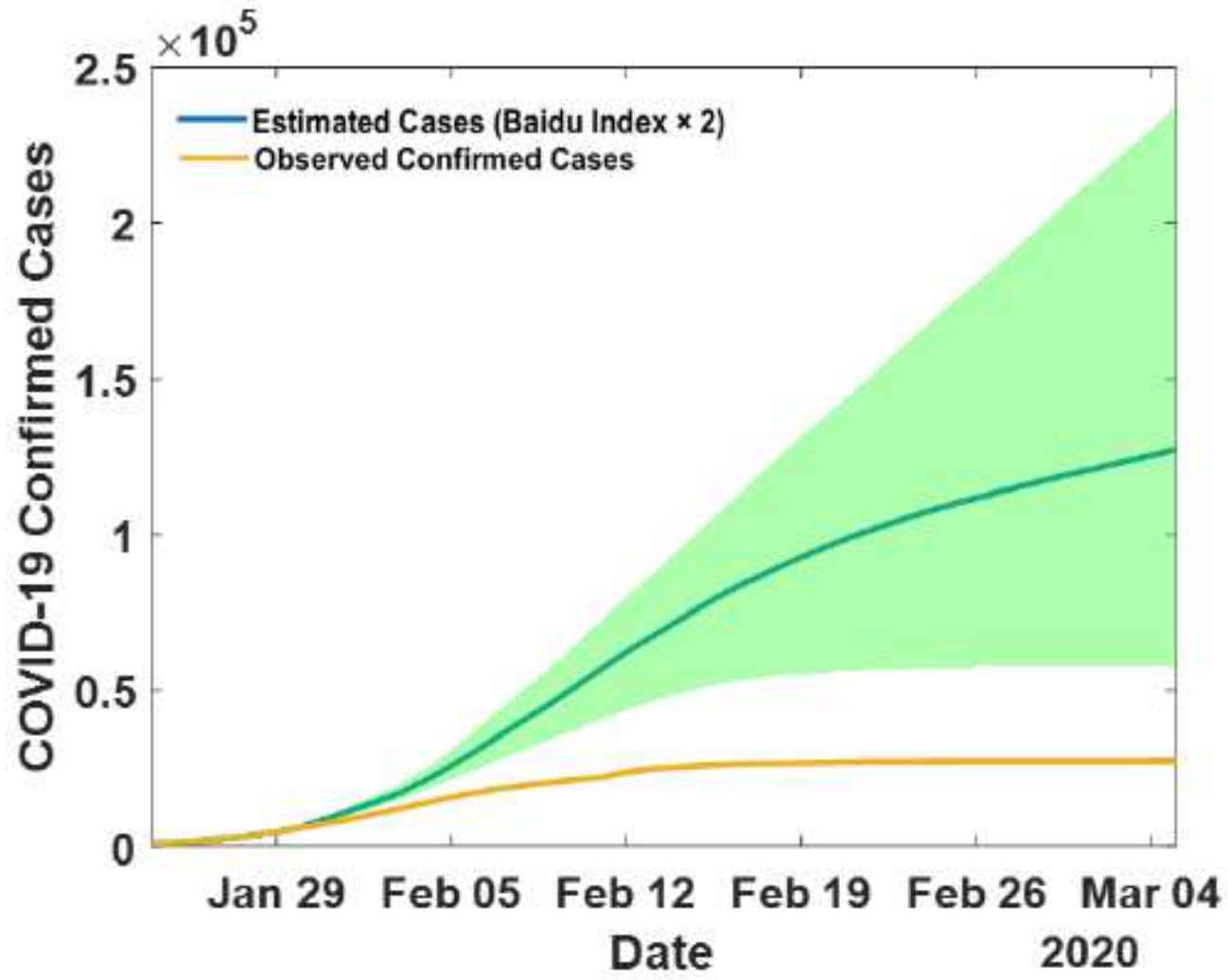

Figure 3 
Estimated number of COVID-19 cases after doubling the Baidu indices for the 100 analyzed cities.
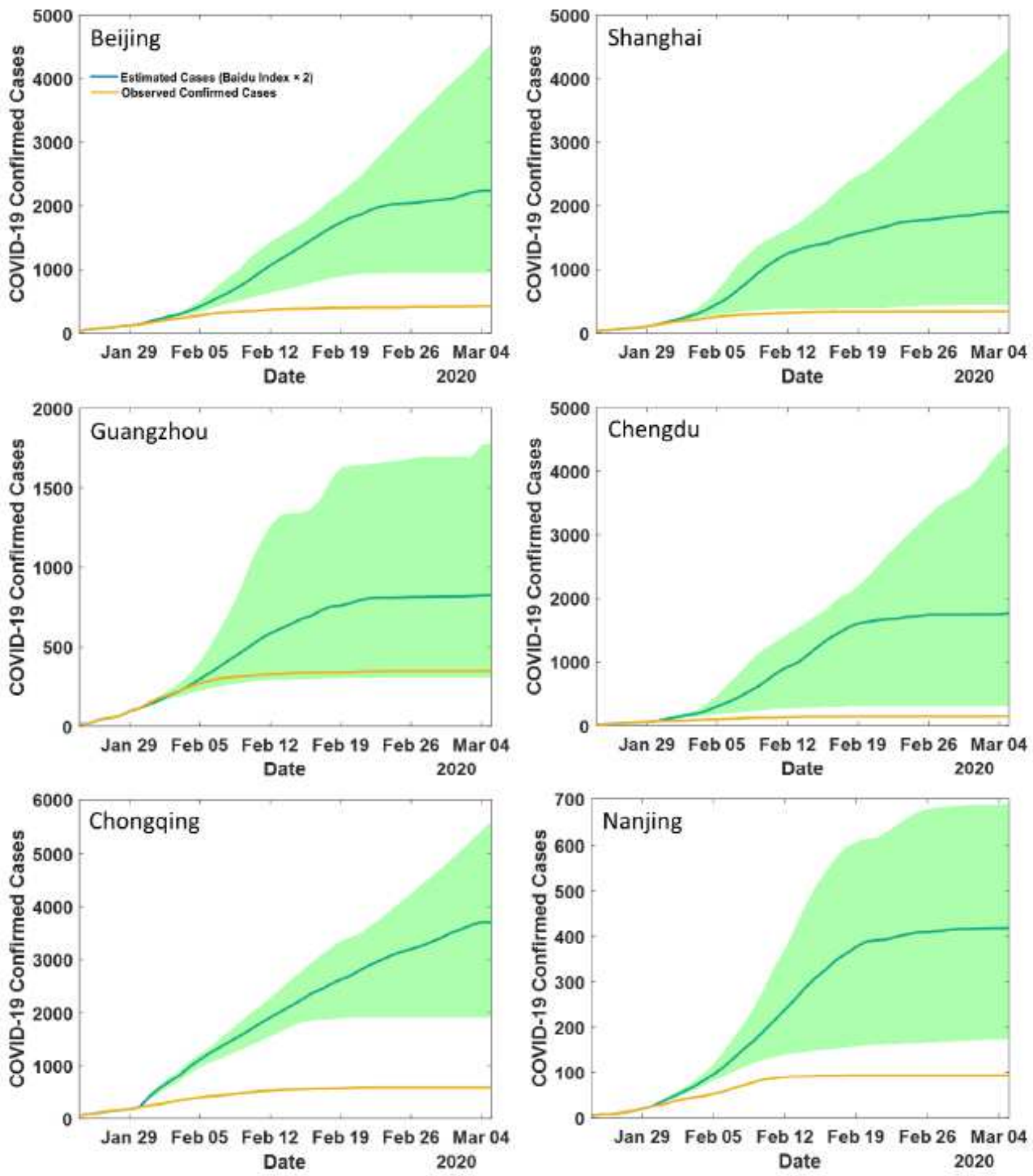

\section{Figure 4}

Estimated number of COVID-19 cases after doubling the Baidu indices for six major cities in China. 

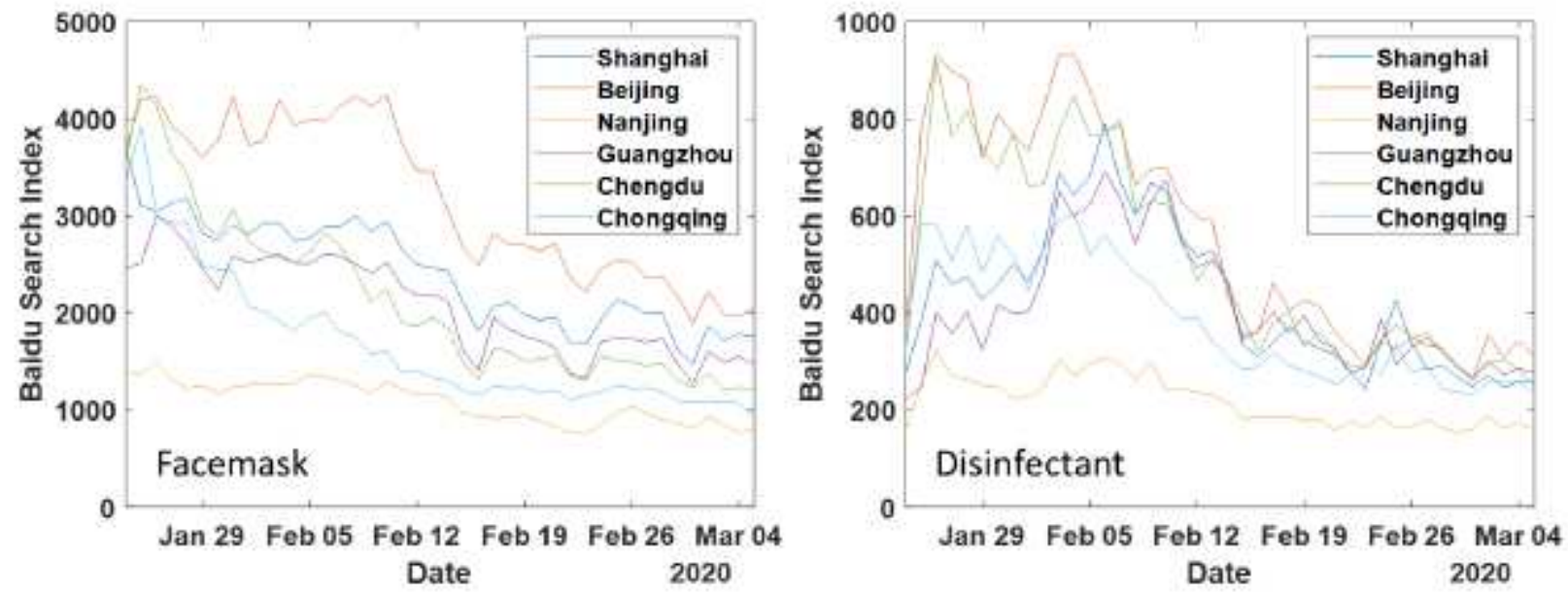

Figure 5

Baidu search indices for "face mask" and "disinfectant" for six major cities in China.
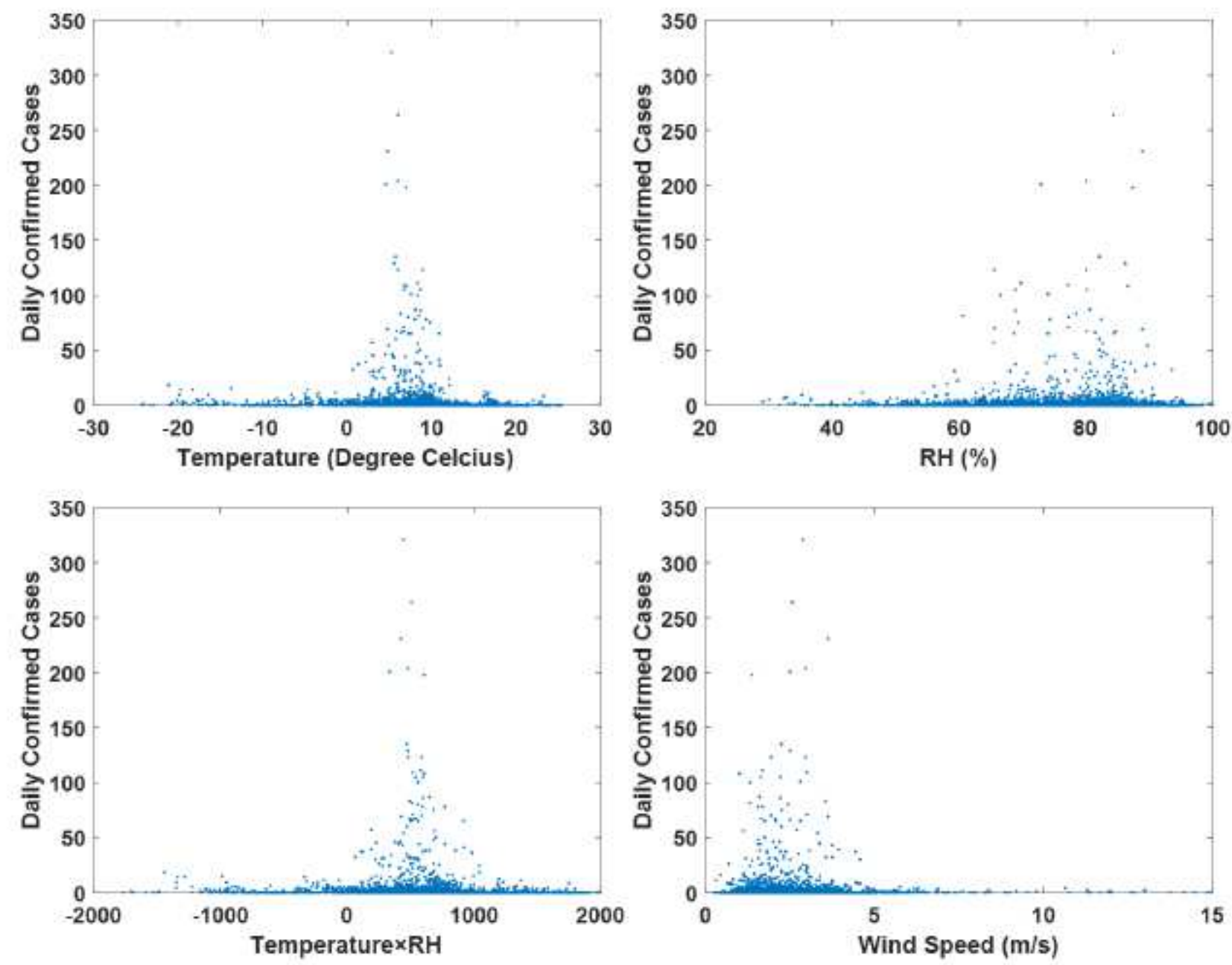

Figure 6 
Relationships between the daily confirmed cases and meteorological factors (previous 4-7 days' averages).

\section{Supplementary Files}

This is a list of supplementary files associated with this preprint. Click to download.

- SupplementalMaterial.pdf 